\title{
An Exploration of the Active Ingredients of Salvia miltiorrhiza in the Treatment of Gastric Cancer and Its Mechanism Based on Network Pharmacology
}

\author{
Xiongdong Zhong \\ Department of General Surgery, Zhuhai People's Hospital (Zhuhai Hospital Affiliated with Jinan University), Zhuhai, P. R. China \\ Email address: \\ zhongxiongdong@sina.com \\ To cite this article: \\ Xiongdong Zhong. An Exploration of the Active Ingredients of Salvia miltiorrhiza in the Treatment of Gastric Cancer and Its Mechanism \\ Based on Network Pharmacology. Journal of Cancer Treatment and Research. Vol. 8, No. 2, 2020, pp. 34-44. \\ doi: $10.11648 /$ j.jctr.20200802.12
}

Received: April 10, 2020; Accepted: May 6, 2020; Published: May 19, 2020

\begin{abstract}
BACKGROUND: Danshen, also known as Salvia miltiorrhiza or radix salvia in Latin, is an important drug whose main pharmacological effects are vasodilation, promotion of blood circulation, and elimination of stasis. In recent years, it has been reported that danshen also has anti-tumor activity. OBJECTIVE: The aim of this study was to explore the feasibility and potential mechanism of S. miltiorrhiza in the treatment of gastric cancer. STUDY DESIGN: We analyzed effective components and target genes of S. miltiorrhiza in the Traditional Chinese Medicine System Pharmacology (TCMSP) database and analysis platform. We then searched the GeneCards database for target genes related to gastric cancer and the intersection of these genes with the active components of $S$. miltiorrhiza. Target genes related to gastric cancer were taken as common potential target genes of $S$. miltiorrhiza, which could act on gastric cancer. Using the R programming language, we drew a Venn map of these common potential target genes. The "component-target gene-disease" network of $S$. miltiorrhiza in the treatment of gastric cancer was established using Cytoscape software version 3.7.1; the protein-protein interaction (PPI) network was constructed in the Search Tool for the Retrieval of Interacting Genes/Proteins (STRING) database. With the help of R and Perl languages, we performed gene ontology (GO) function and Kyoto Encyclopedia of Genes and Genomes (KEGG) pathway enrichment analyses of potential target genes of $S$. miltiorrhiza in the treatment of gastric cancer. RESULTS: We extracted a total of 65 active components from $S$. miltiorrhiza, including dihydrotanshinone I and miltionones I and II, as well as 102 potential target genes for gastric cancer. According to the Degree ranking in Cytoscape3.7.1 software, the top 10 potential target genes were protein kinase B1 (AKT1), interleukin-6 (IL-6), vascular endothelial growth factor A (VEGFA), epidermal growth factor receptor $(E G F R)$, Fos, mitogenactivated protein kinase 1 (MAPK1), Myc, JUN, Caspase-3 (CASP3), and signal transducer and activator of transcription 3 (STAT3). Pathway enrichment mainly involved signaling pathways such as phosphoinositide 3-kinase (PI3K)-Akt, hypoxiainducible factor 1 (HIF-1), and IL-17. CONCLUSION: Based on network pharmacology, S. miltiorrhiza is expected to be mined as a candidate Traditional Chinese Medicine (TCM) for the treatment of gastric cancer. Its mechanism for treating this cancer operates via multiple components and pathways. This study provides the basic theory and the basis for further research.
\end{abstract}

Keywords: Salvia miltiorrhiza, Gastric Cancer, Network Pharmacology, Target Gene, GO Function Enrichment Analysis, KEGG Pathway Enrichment Analysis

\section{Introduction}

Gastric cancer is the second-leading cause of cancerrelated deaths worldwide, with more than half of cases occurring in East Asia [1]. In the United States, about 28,000 new cases and 10,960 deaths occurred in 2017 [2]. In China, gastric cancer is the second-leading cause of cancer-related deaths, with an estimated 500,000 deaths per year [3]. Despite improvements in diagnosis and treatment, the longterm survival rate of many gastric-cancer patients is still very low, and the 5-year survival rate is $<20 \%$ [4]. Most patients with gastric cancer already had tumor metastasis at diagnosis; the high rate of tumor metastasis leads to the low survival rate [5]. The etiology and pathogenesis of gastric cancer have 
not been fully elucidated. Therefore, it is urgent for doctors to find strategies to improve the survival rate of gastriccancer patients.

In recent years, Traditional Chinese Medicine (TCM) has played an increasingly important role in the prevention and treatment of diseases for Chinese people, Asians in general, and even people in other countries [6]. This is because it has good therapeutic effects and a low rate of side effects. Therefore, TCM has attracted more and more attention worldwide. However, the lack of reliable evidence for its effectiveness and safety might be an important reason for its difficulties in being recognized as legitimate treatment in Europe and the US [7].

The network pharmacology method, which integrates system biology with computer technology, could provide strategies for research into the mechanisms of TCM [8]. In the past few years, hundreds of network pharmacology studies have confirmed the molecular mechanisms of TCM [9-11]. Danshen, also known as Salvia miltiorrhiza or radix salvia in Latin, is an important TCM drug. Its main pharmacological effects are vasodilation, promotion of blood circulation, and elimination of stasis [12]. One meta-analysis of high-quality randomized controlled trials found that $S$. miltiorrhiza could improve the clinical prognosis of gastriccancer patients [13], but the molecular mechanism by which this occurred has not been clarified. In this study, we used network pharmacology to elucidate the feasibility and potential pharmacological mechanism of $S$. miltiorrhiza in the treatment of gastric cancer.

\section{Materials and Methods}

All researches were approved by the Research Committee of Zhuhai People's Hospital (Zhuhai Hospital Affiliated with Jinan University), and carried out in accordance with the approved guidelines.

\subsection{Searching Effective Components of S. miltiorrhiza}

We input the keyword "Salvia miltiorrhiza" into the Traditional Chinese Medicine System Pharmacology (TCMSP) database and analysis platform and retrieved $S$. miltiorrhiza's active ingredients. According to recommendations in the literature, we regarded compounds with oral bioavailability (OB) $>30 \%$ and drug-likeness (DL) $>0.18$ as effective components.

\subsection{Searching Potential Target Genes of S. miltiorrhiza}

We searched all target genes of $S$. miltiorrhiza and then extracted the target genes of its effective components. We imported 102 potential target genes of S. miltiorrhiza's active components into the UniProt database (https://www.uniprot.org/), selected "human" as the species, and obtained names of target genes and their corresponding acronyms.

\subsection{Searching for Gastric-cancer-related Genes}

The GeneCards database (http://www.genecards.org/) is a 1-stop database for human-gene annotation that covers nearly $90 \%$ of human protein-coding genes. We input the keyword "gastric cancer" into GeneCards and then exported the retrieval results.

\subsection{Intersection of Potential Target Genes of S. miltiorrhiza and Gastric-cancer-related Genes}

We considered genes that were both potential target genes of $S$. miltiorrhiza and gastric-cancer-related genes to be the genes of interaction between $S$. miltiorrhiza and gastric cancer.

\subsection{Constructing a Component-target Gene-disease Network}

We deleted effective components that could not act on gastric-cancer-related target genes. Then we imported the remaining effective components and potential target genes for gastric-cancer treatment into Cytoscape software version 3.7.1 (US National Institute of General Medical Sciences [NIGMS], Bethesda, Maryland, US; https://cytoscape.org/) to build an effective component-target gene-disease network.

\subsection{Constructing a Protein-protein Interaction (PPI) Network}

We input potential target genes of S. miltiorrhiza in the treatment of gastric cancer into the Search Tool for the Retrieval of Interacting Genes/Proteins (STRING) database (https://string-db.org/) and selected "Homo sapiens" as the species. We obtained the PPI network in tab-separated values (.tsv) file format; the PPI network picture was saved in Portable Network Graphics (.png) format.

\subsection{Screening Core Genes of PPI Network}

Using the $\mathrm{R}$ programming language, we calculated a genes.tsv file and selected the top 30 target genes of degree value as the core genes.

\subsection{Gene Ontology (GO) Function and Kyoto Encyclopedia of Genes and Genomes (KEGG) Pathway Enrichment Analyses}

Using $\mathrm{R}$ language, we analyzed our GO function enrichment analysis of potential target genes and our KEGG pathway enrichment analysis, including biological processes, molecular functions, and cell components. We selected the path whose kinase insert domain receptor (KDR) value (i.e., $Q$-value) was $<0.05$, and then we ranked the paths with the 20 highest KDR values in order to draw a histogram and bubble chart.

\section{Results}

\subsection{Acquisition of Effective Components and Potential Target Genes of S. miltiorrhiza}

From the TCMSP database we obtained 65 active components of $S$. miltiorrhiza, such as dihydrotanshinone I 
and miltionones I and II, using the screening conditions of converted target genes corresponding to active components $\mathrm{OB}>30 \%$ and $\mathrm{DL}>0.18$ (Table 1). At the same time, we of S. miltiorrhiza in the UniProt database.

Table 1. Active components of Salvia miltiorrhiza.

\begin{tabular}{|c|c|c|c|c|c|}
\hline No. & molID & molName & MW & OB (\%) & DL \\
\hline 01 & MOL001601 & 1,2,5,6-Tetrahydrotanshinone & 280.34 & 38.75 & 0.36 \\
\hline 02 & MOL001659 & Poriferasterol & 412.77 & 43.83 & 0.76 \\
\hline 03 & MOL001771 & Poriferast-5-en-3beta-ol & 414.79 & 36.91 & 0.75 \\
\hline 04 & MOL001942 & Isoimperatorin & 270.3 & 45.46 & 0.23 \\
\hline 05 & MOL002222 & Sugiol & 300.48 & 36.11 & 0.28 \\
\hline 06 & MOL002651 & Dehydrotanshinone II A & 292.35 & 43.76 & 0.4 \\
\hline 07 & MOL002776 & Baicalin & 446.39 & 40.12 & 0.75 \\
\hline 08 & MOL000569 & Digallate & 322.24 & 61.85 & 0.26 \\
\hline 09 & MOL000006 & Luteolin & 286.25 & 36.16 & 0.25 \\
\hline 10 & MOL006824 & $\alpha$-Amyrin & 426.8 & 39.51 & 0.76 \\
\hline 11 & MOL007036 & 5,6-Dihydroxy-7-isopropyl-1,1-dimethyl-2,3-dihydrophenanthren-4-one & 298.41 & 33.77 & 0.29 \\
\hline 12 & MOL007041 & 2-Isopropyl-8-methylphenanthrene-3,4-dione & 264.34 & 40.86 & 0.23 \\
\hline 13 & MOL007045 & 3 $\alpha$-Hydroxytanshinone II a & 310.37 & 44.93 & 0.44 \\
\hline 14 & MOL007048 & (E)-3-[2-(3,4-Dihydroxyphenyl)-7-hydroxy-benzofuran-4-yl]acrylic acid & 312.29 & 48.24 & 0.31 \\
\hline 15 & MOL007049 & 4-Methylenemiltirone & 266.36 & 34.35 & 0.23 \\
\hline 16 & MOL007050 & 2-(4-hydroxy-3-methoxyphenyl)-5-(3-hydroxypropyl)-7-methoxy-3-benzofurancarboxaldehyde & 356.4 & 62.78 & 0.4 \\
\hline 17 & MOL007051 & 6-o-Syringyl-8-o-acetyl shanzhiside methyl ester & 628.64 & 46.69 & 0.71 \\
\hline 18 & MOL007058 & Formyltanshinone & 290.28 & 73.44 & 0.42 \\
\hline 19 & MOL007059 & 3-beta-Hydroxymethyllenetanshiquinone & 294.32 & 32.16 & 0.41 \\
\hline 20 & MOL007061 & Methylenetanshinquinone & 278.32 & 37.07 & 0.36 \\
\hline 21 & MOL007063 & Przewalskin a & 398.49 & 37.11 & 0.65 \\
\hline 22 & MOL007064 & Przewalskin $b$ & 330.46 & 110.32 & 0.44 \\
\hline 23 & MOL007068 & Przewaquinone B & 292.3 & 62.24 & 0.41 \\
\hline 24 & MOL007069 & Przewaquinone c & 296.34 & 55.74 & 0.4 \\
\hline 25 & MOL007070 & (6S,7R)-6,7-dihydroxy-1,6-dimethyl-8,9-dihydro-7H-naphtho[8,7-g]benzofuran-10,11-dione & 312.34 & 41.31 & 0.45 \\
\hline 26 & MOL007071 & Przewaquinone $\mathrm{f}$ & 312.34 & 40.31 & 0.46 \\
\hline 27 & MOL007077 & Sclareol & 308.56 & 43.67 & 0.21 \\
\hline 28 & MOL007079 & Tanshinaldehyde & 308.35 & 52.47 & 0.45 \\
\hline 29 & MOL007081 & Danshenol B & 354.48 & 57.95 & 0.56 \\
\hline 30 & MOL007082 & Danshenol A & 336.41 & 56.97 & 0.52 \\
\hline 31 & MOL007085 & Salvilenone & 292.4 & 30.38 & 0.38 \\
\hline 32 & MOL007088 & Cryptotanshinone & 296.39 & 52.34 & 0.4 \\
\hline 33 & MOL007093 & Dan-shexinkum d & 336.41 & 38.88 & 0.55 \\
\hline 34 & MOL007094 & Danshenspiroketallactone & 282.36 & 50.43 & 0.31 \\
\hline 35 & MOL007098 & Deoxyneocryptotanshinone & 298.41 & 49.4 & 0.29 \\
\hline 36 & MOL007100 & Dihydrotanshinlactone & 266.31 & 38.68 & 0.32 \\
\hline 37 & MOL007101 & Dihydrotanshinone I & 278.32 & 45.04 & 0.36 \\
\hline 38 & MOL007105 & Epidanshenspiroketallactone & 284.38 & 68.27 & 0.31 \\
\hline 39 & MOL007107 & C09092 & 286.5 & 36.07 & 0.25 \\
\hline 40 & MOL007108 & Isocryptotanshi-none & 296.39 & 54.98 & 0.39 \\
\hline 41 & MOL007111 & Isotanshinone II & 294.37 & 49.92 & 0.4 \\
\hline 42 & MOL007115 & Manool & 304.57 & 45.04 & 0.2 \\
\hline 43 & MOL007118 & Microstegiol & 298.46 & 39.61 & 0.28 \\
\hline 44 & MOL007119 & Miltionone I & 312.39 & 49.68 & 0.32 \\
\hline 45 & MOL007120 & Miltionone II & 312.39 & 71.03 & 0.44 \\
\hline 46 & MOL007121 & Miltipolone & 300.43 & 36.56 & 0.37 \\
\hline 47 & MOL007122 & Miltirone & 282.41 & 38.76 & 0.25 \\
\hline 48 & MOL007123 & Miltirone II & 272.32 & 44.95 & 0.24 \\
\hline 49 & MOL007124 & Neocryptotanshinone II & 270.35 & 39.46 & 0.23 \\
\hline 50 & MOL007125 & Neocryptotanshinone & 314.41 & 52.49 & 0.32 \\
\hline 51 & MOL007127 & 1-methyl-8,9-dihydro-7H-naphtho[5,6-g]benzofuran-6,10,11-trione & 280.29 & 34.72 & 0.37 \\
\hline 52 & MOL007130 & Prolithospermic acid & 314.31 & 64.37 & 0.31 \\
\hline 53 & MOL007132 & (2R)-3-(3,4-dihydroxyphenyl)-2-[(Z)-3-(3,4-dihydroxyphenyl)acryloyl]oxy-propionic acid & 360.34 & 109.38 & 0.35 \\
\hline 54 & MOL007140 & (Z)-3-[2-[(E)-2-(3,4-dihydroxyphenyl)vinyl]-3,4-dihydroxy-phenyl]acrylic acid & 314.31 & 88.54 & 0.26 \\
\hline 55 & MOL007141 & Salvianolic acid g & 340.3 & 45.56 & 0.61 \\
\hline
\end{tabular}




\begin{tabular}{|c|c|c|c|c|c|}
\hline No. & mollD & molName & MW & OB (\%) & DL \\
\hline 56 & MOL007142 & Salvianolic acid $\mathrm{j}$ & 538.49 & 43.38 & 0.72 \\
\hline 57 & MOL007143 & Salvilenone I & 270.40 & 32.43 & 0.23 \\
\hline 58 & MOL007145 & Salviolone & 268.38 & 31.72 & 0.24 \\
\hline 59 & MOL007149 & NSC 122421 & 300.48 & 34.49 & 0.28 \\
\hline 60 & MOL007150 & (6S)-6-hydroxy-1-methyl-6-methylol-8,9-dihydro-7H-naphtho[8,7-g]benzofuran-10,11-quinone & 312.34 & 75.39 & 0.46 \\
\hline 61 & MOL007151 & Tanshindiol B & 312.34 & 42.67 & 0.45 \\
\hline 62 & MOL007152 & Przewaquinone E & 312.34 & 42.85 & 0.45 \\
\hline 63 & MOL007154 & Tanshinone iia & 294.37 & 49.89 & 0.4 \\
\hline 64 & MOL007155 & (6S)-6-(hydroxymethyl)-1,6-dimethyl-8,9-dihydro-7H-naphtho[8,7-g]benzofuran-10,11-dione & 310.37 & 65.26 & 0.45 \\
\hline 65 & MOL007156 & Tanshinone VI & 296.34 & 45.64 & 0.30 \\
\hline
\end{tabular}

There are 65 active components of $\mathrm{S}$. miltiorrhiza. molID=molecular identification number; molName=molecular name; $\mathrm{MW}=\mathrm{molecular}$ weight; $\mathrm{OB}=$ oral bioavailability; $\mathrm{DL}=$ drug-likeness.

\subsection{Predicting Potential Target Genes of S. miltiorrhiza in the Treatment of Gastric Cancer}

We retrieved a total of 18,221 gastric-cancer-related genes from the GeneCards database and compared them with target genes corresponding to each effective component of S. miltiorrhiza. We selected 102 common target genes (Table 2) as potential target genes of S. miltiorrhiza in the treatment of gastric cancer (Figure 1).

Table 2. Potential target information of S. miltiorrhiza.

\begin{tabular}{|c|c|c|c|c|c|c|c|c|}
\hline No. & Uniprotkb & Target & No. & Uniprotkb & Target & No. & Uniprotkb & Target \\
\hline 1 & P22303 & $A C H E$ & 35 & P21918 & DRD5 & 69 & P01106 & $M Y C$ \\
\hline 2 & Q08462 & $A D C Y 2$ & 36 & P42892 & ECE1 & 70 & Q15788 & NCOA1 \\
\hline 3 & P35348 & $A D R A 1 A$ & 37 & P14138 & $E D N 3$ & 71 & P25963 & NFKBIA \\
\hline 4 & P35368 & $A D R A 1 B$ & 38 & P25101 & $E D N R A$ & 72 & P60321 & NOS2 \\
\hline 5 & P08913 & $A D R A 2 A$ & 39 & P00533 & $E G F R$ & 73 & P06748 & NPM1 \\
\hline 6 & P18089 & $A D R A 2 B$ & 40 & P04626 & $E R B B 2$ & 74 & O75469 & NR1I2 \\
\hline 7 & P18825 & $A D R A 2 C$ & 41 & P03372 & ESR1 & 75 & P04150 & $\mathrm{NR} 3 \mathrm{Cl}$ \\
\hline 8 & P07550 & $A D R B 2$ & 42 & Q92731 & ESR2 & 76 & P08235 & $N R 3 C 2$ \\
\hline 9 & O95433 & AHSAl & 43 & P08709 & $F 7$ & 77 & Q9BZD4 & $N U F 2$ \\
\hline 10 & $\mathrm{P} 15121$ & $A K R 1 B 1$ & 44 & P49327 & FASN & 78 & P41143 & OPRD1 \\
\hline 11 & P31749 & $A K T 1$ & 45 & P01100 & FOS & 79 & P35372 & OPRMI \\
\hline 12 & P05067 & $A P P$ & 46 & P78334 & GABRE & 80 & Q9UKK3 & PARP4 \\
\hline 13 & P10275 & $A R$ & 47 & P49841 & GSK3B & 81 & P12004 & PCNA \\
\hline 14 & P10415 & $B C L 2$ & 48 & P09211 & GSTP1 & 82 & P06401 & $P G R$ \\
\hline 15 & Q07817 & $B C L 2 L 1$ & 49 & P09601 & $H M O X 1$ & 83 & P37231 & $P P A R G$ \\
\hline 16 & O15392 & BIRC5 & 50 & P46098 & $H T R 3 A$ & 84 & P07477 & PRSS1 \\
\hline 17 & P30988 & $C A L C R$ & 51 & P05362 & ICAMI & 85 & O14684 & PTGES \\
\hline 18 & P42574 & CASP3 & 52 & P01579 & $I F N G$ & 86 & P23219 & PTGS1 \\
\hline 19 & P55210 & CASP7 & 53 & P22301 & IL10 & 87 & P35354 & PTGS2 \\
\hline 20 & P55211 & CASP9 & 54 & P60568 & $I L 2$ & 88 & P06400 & $R B 1$ \\
\hline 21 & P20248 & CCNA2 & 55 & P05112 & IL4 & 89 & Q04206 & $R E L A$ \\
\hline 22 & P14635 & $C C N B 1$ & 56 & P05231 & IL6 & 90 & P19793 & $R X R A$ \\
\hline 23 & P24385 & $C C N D 1$ & 57 & P06213 & $I N S R$ & 91 & Q14524 & SCN5A \\
\hline 24 & P29965 & $C D 40 L G$ & 58 & P05106 & $I T G B 3$ & 92 & P14672 & $S L C 2 A 4$ \\
\hline 25 & P38936 & $C D K N 1 A$ & 59 & P05412 & $J U N$ & 93 & P23975 & $S L C 6 A 2$ \\
\hline 26 & O14757 & CHEK1 & 60 & Q12809 & $\mathrm{KCNH} 2$ & 94 & Q01959 & SLC6A3 \\
\hline 27 & P11229 & CHRMI & 61 & P28482 & MAPK1 & 95 & P31645 & SLC6A4 \\
\hline 28 & P08172 & CHRM2 & 62 & Q16539 & MAPK14 & 96 & P40763 & STAT3 \\
\hline 29 & P20309 & CHRM3 & 63 & Q07820 & $M C L 1$ & 97 & O95150 & TNFSF 15 \\
\hline 30 & Q15822 & CHRNA2 & 64 & Q00987 & $M D M 2$ & 98 & P11387 & TOP1 \\
\hline 31 & P04798 & CYP1A1 & 65 & P08581 & $M E T$ & 99 & P11388 & TOP $2 A$ \\
\hline 32 & P05177 & CYP1A2 & 66 & P03956 & $M M P 1$ & 100 & Q9H3D4 & TP63 \\
\hline 33 & P08684 & СУР $3 A 4$ & 67 & P08253 & $M M P 2$ & 101 & P14679 & $T Y R$ \\
\hline 34 & P14416 & DRD2 & 68 & P14780 & $M M P 9$ & 102 & $\mathrm{P} 15692$ & VEGFA \\
\hline
\end{tabular}

There are 102 target genes corresponding to active components of S. miltiorrhiza in the UniProt database. 


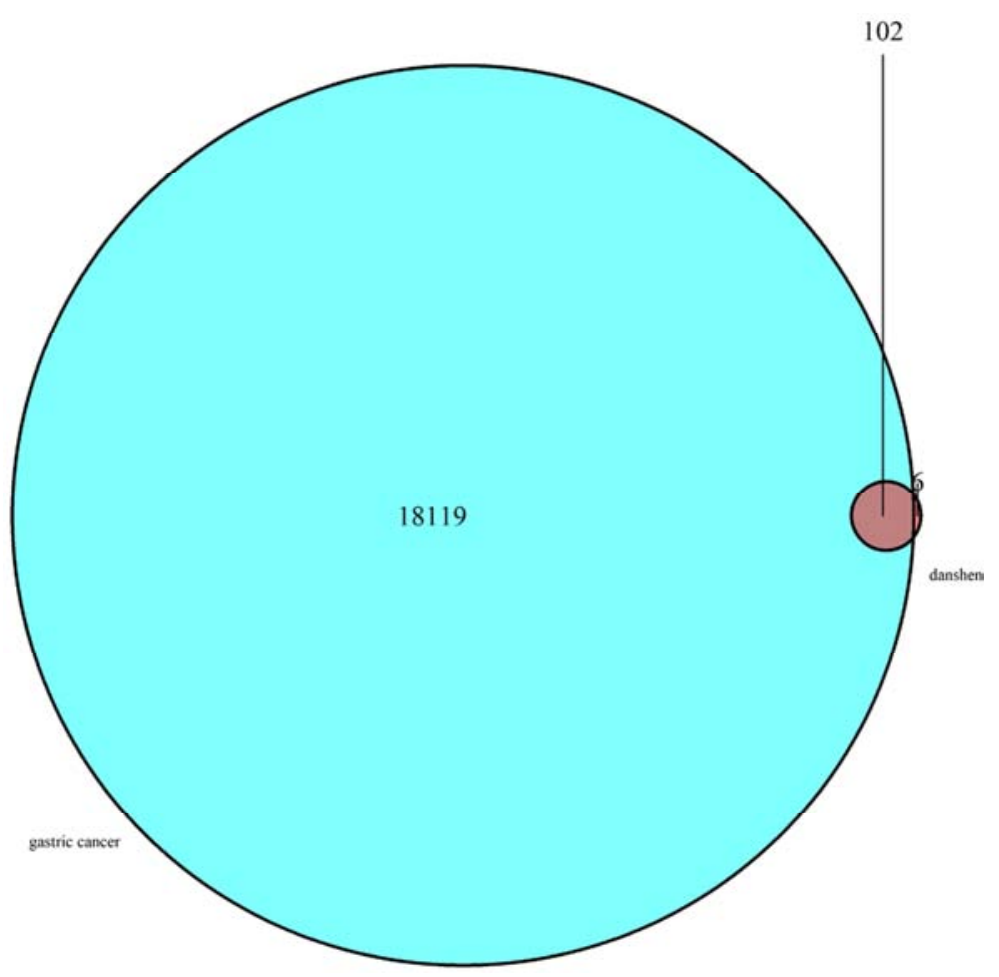

Figure 1. The target genes in common between S. miltiorrhiza and gastric cancer.

\subsection{Constructing a Component-target Gene-disease Network}

We introduced potential target genes of $S$. miltiorrhiza for gastric-cancer treatment into Cytoscape to construct a component-target gene-disease network (Figure 2). The network had 102 nodes and 1190 strip edges. In Figure 2, the blue octagonal shape represents $S$. miltiorrhiza, the purple triangle represents the active-component molecule, the green circle represents the target gene, and the red diamond represents the disease. As the figure shows, the same active ingredient can correspond to different target genes, and different active ingredients can correspond to the same target gene. This suggested that the potential mechanism of $S$. miltiorrhiza in the treatment of gastric cancer is mediated by multi-component and multi-target genes.

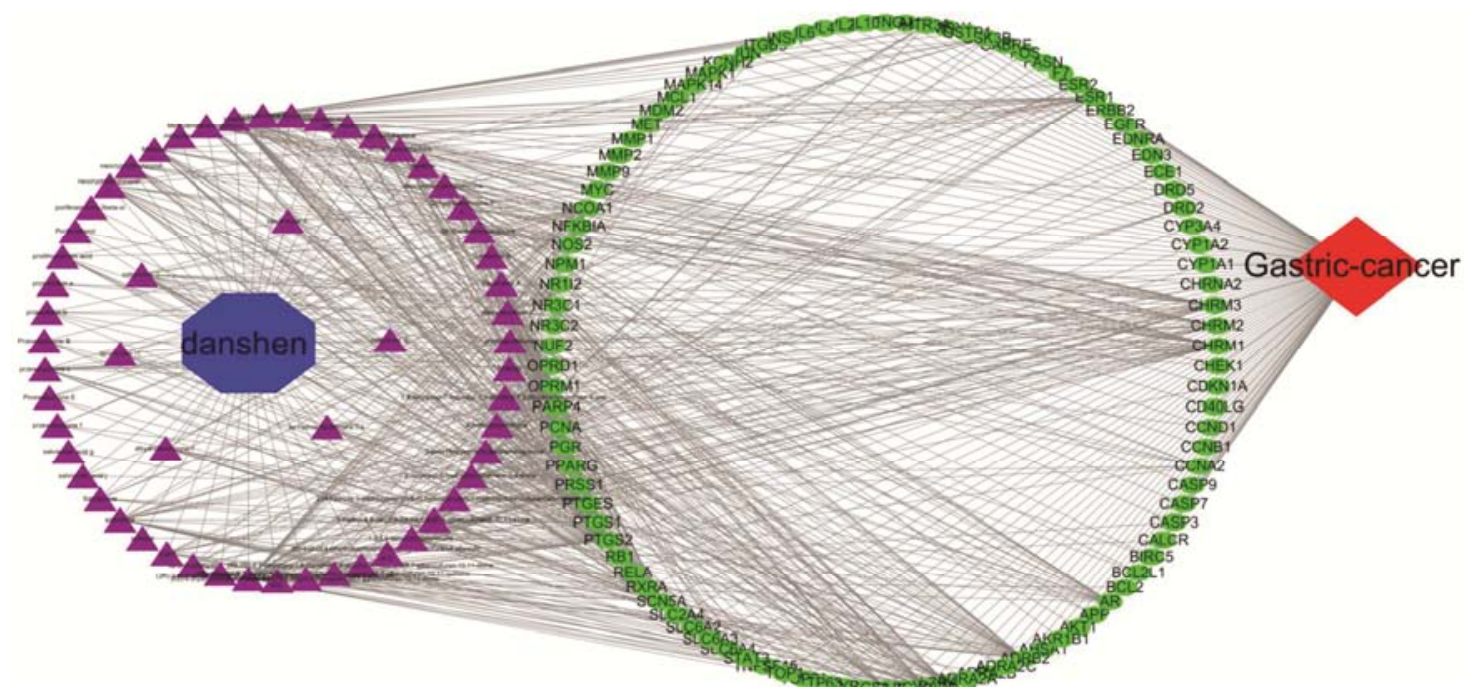

Figure 2. Construction of component-target gene-disease network.

\subsection{Constructing a PPI Network and Screening Core Target Genes}

We imported potential target genes of S. miltiorrhiza in gastric-cancer treatment into the STRING database and obtained a PPI network (Figure 3) and TSV file. With the help of R language, we calculated a genes.tsv file and obtained the top 30 target genes of degree value (Figure 4). 


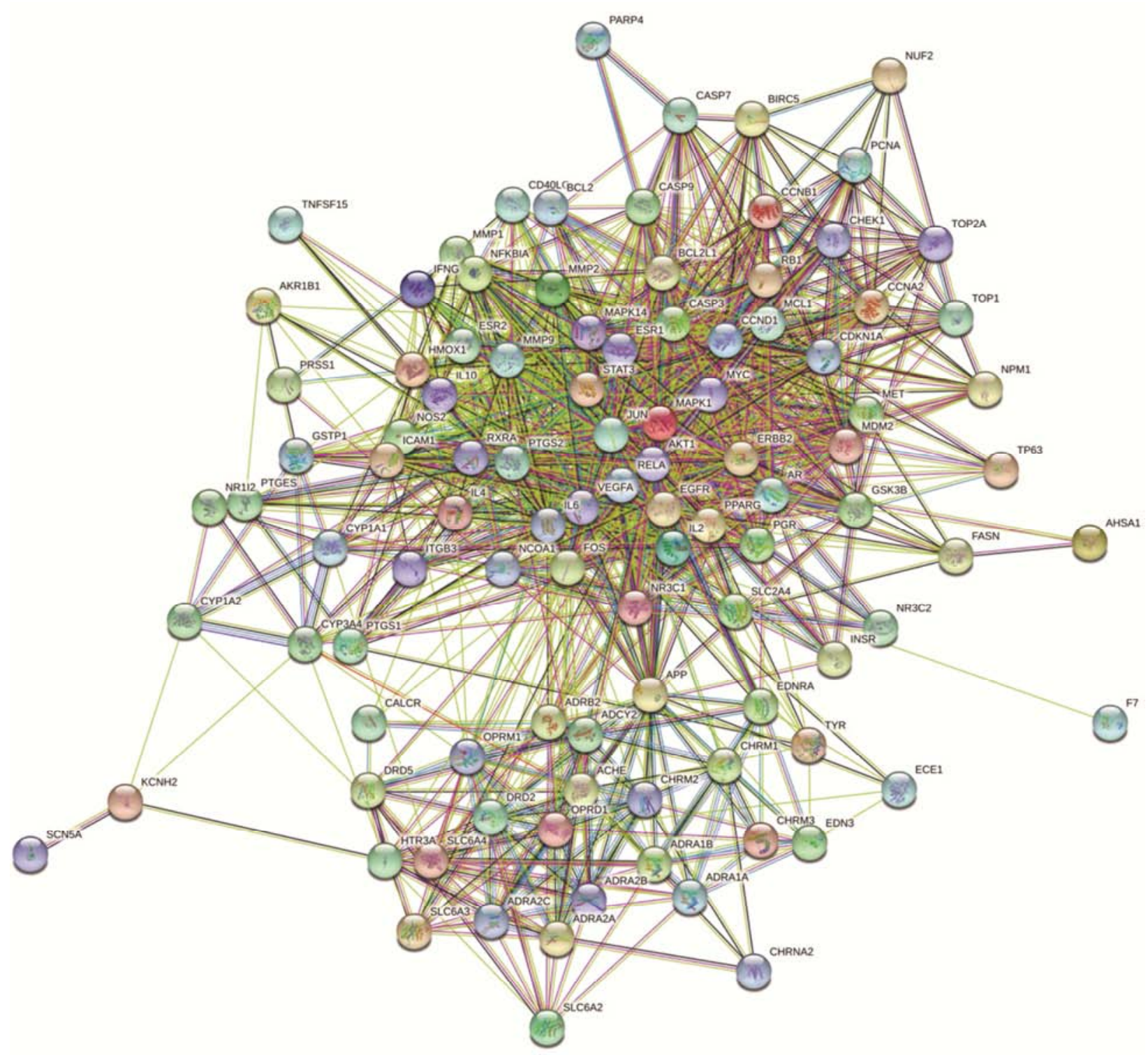

Figure 3. Construction of PPI network.

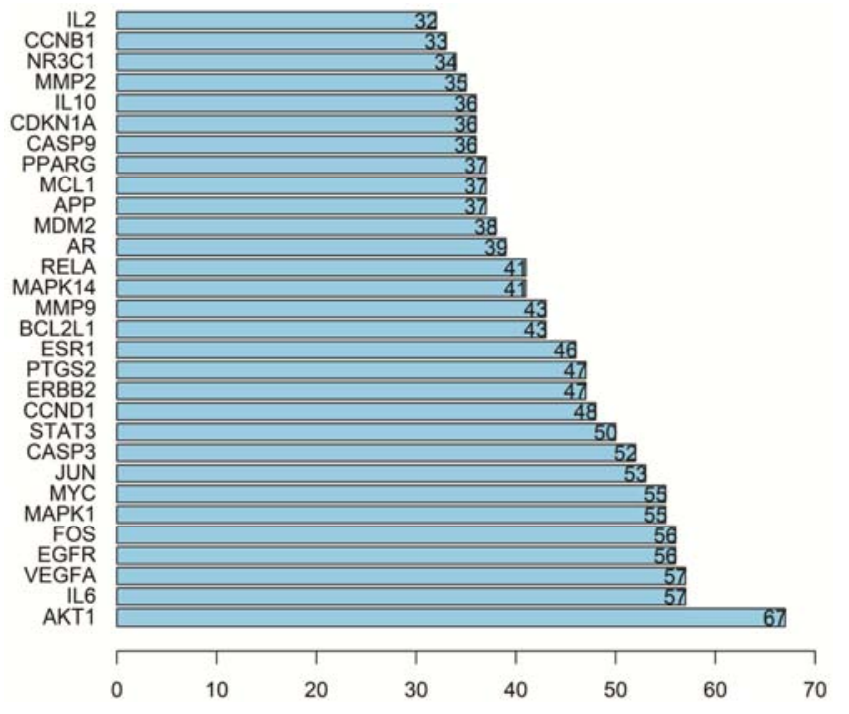

Figure 4. Top 30 core target genes.

\subsection{GO Function and KEGG Pathway Enrichment Analyses}

Using R language, we performed GO function and KEGG pathway enrichment analyses of potential target genes of $S$. miltiorrhiza in gastric-cancer treatment. The results of GO function enrichment analysis (Figures 5 and 6) showed that the biological processes were mainly those such as transcription factor activity, direct ligand-regulated sequencespecific DNA binding, and activating transcription factor binding. The cell components were mainly those such as nuclear receptor activity and chromatin binding, while the molecular functions were mainly those such as steroid hormone receptor activity and catecholamine binding. The results of KEGG pathway enrichment analysis (Figures 7 and 8) showed that potential target genes of S. miltiorrhiza in the treatment of gastric cancer were mainly enriched in signaling pathways such as PI3K-Akt, HIF-1, and IL-17. 


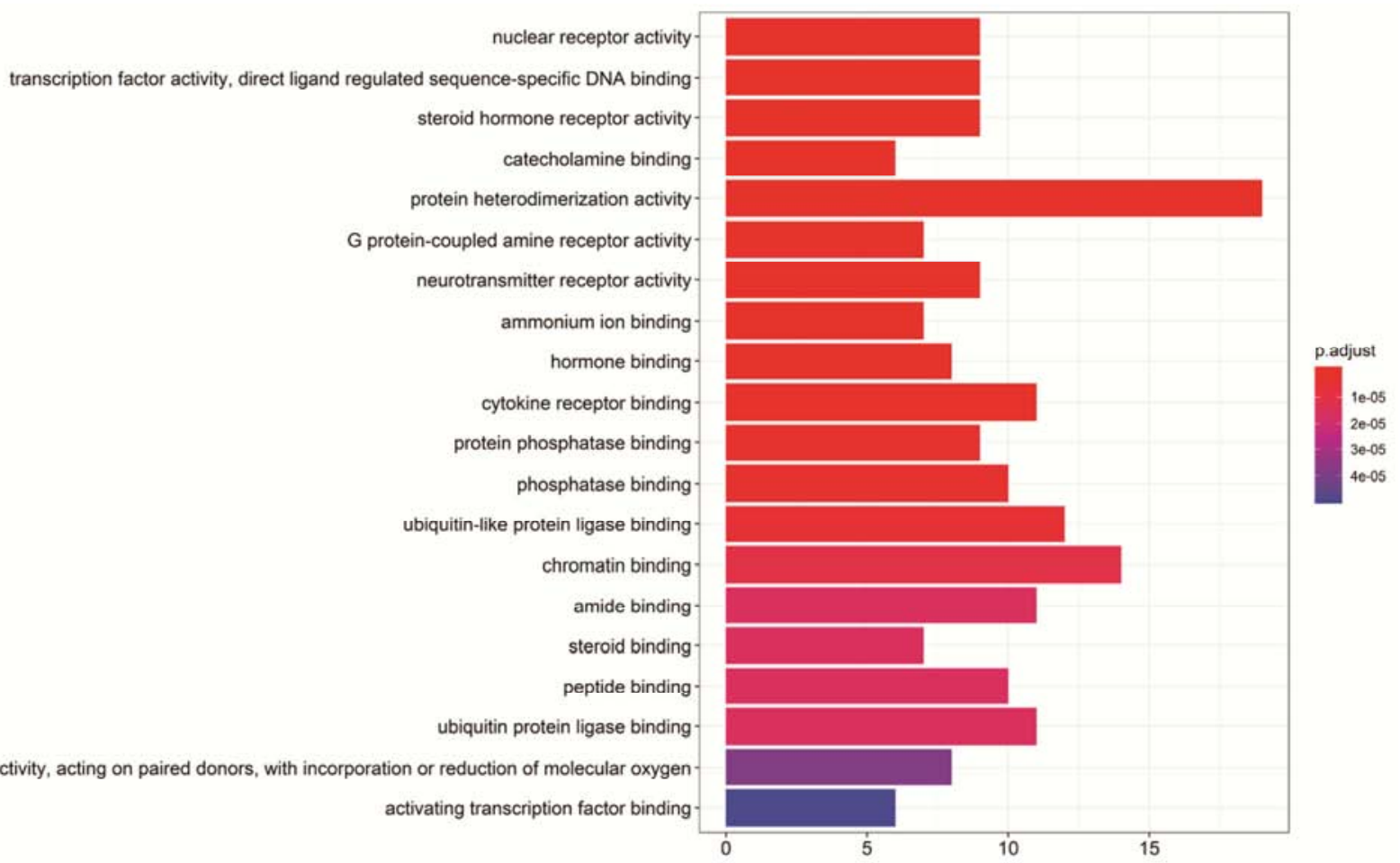

Figure 5. GO functional enrichment analysis (histogram).

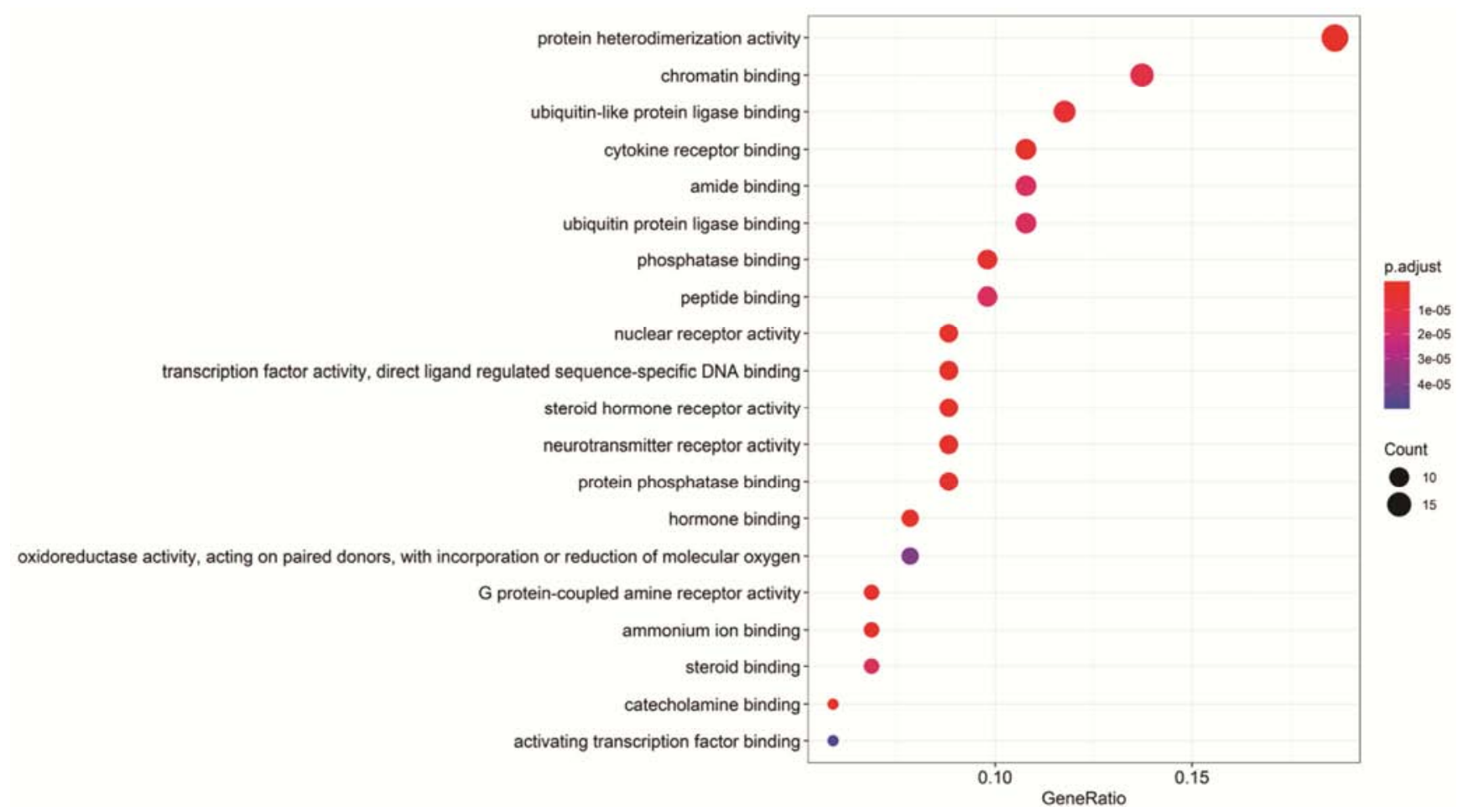

Figure 6. GO functional enrichment analysis (bubble chart). 


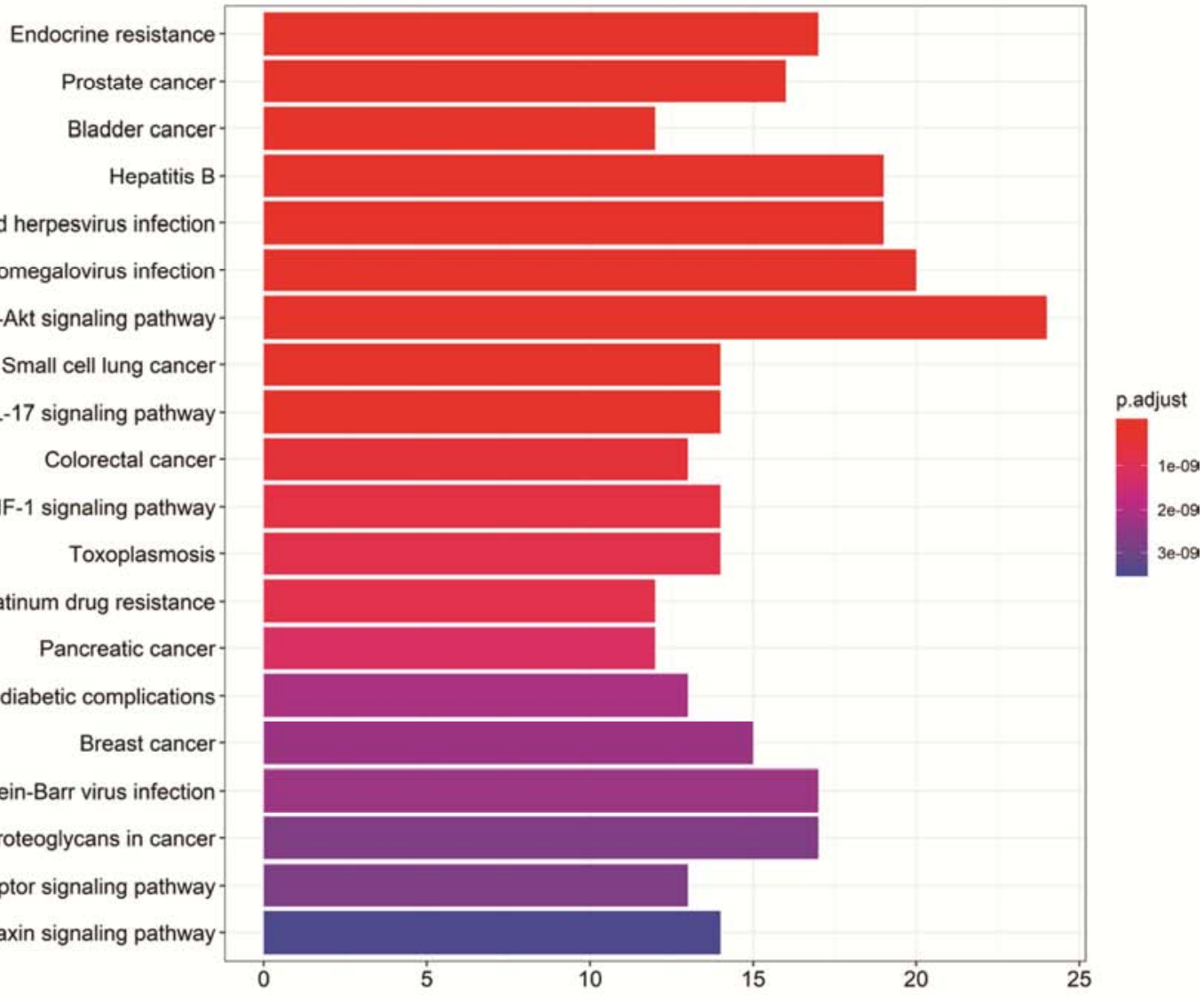

Figure 7. Results of KEGG pathway enrichment analysis (histogram).

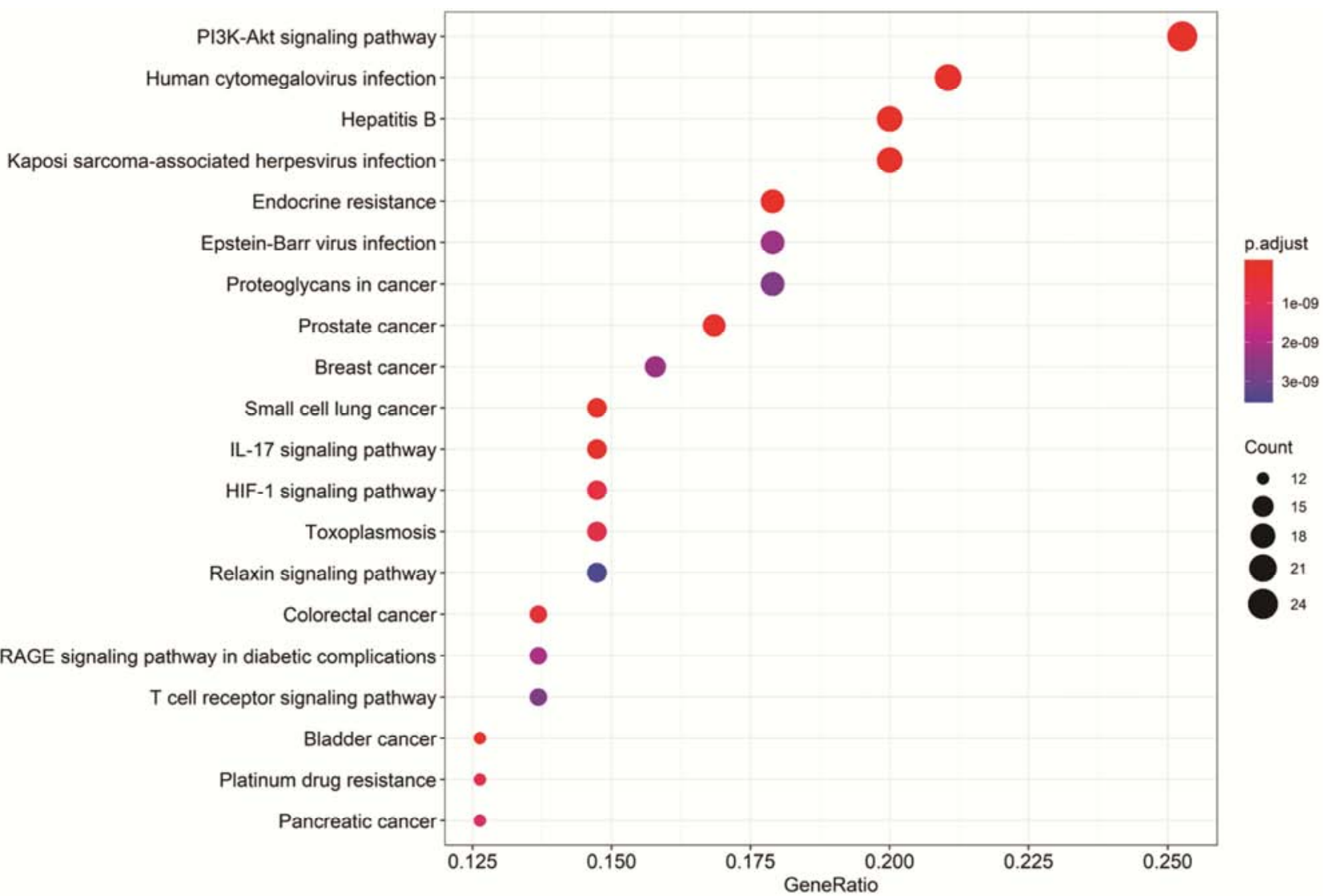

Figure 8. Results of KEGG pathway enrichment analysis (bubble chart). 


\section{Discussion}

TCM, an important auxiliary and alternative medical method [14], has a history dating back thousands of years and has been popularized in many countries. Modern research shows that the effective ingredients in TCM could become candidates for anti-tumor drugs. TCM has unique advantages [15]: it employs compound drugs with multitarget and multi-component effects. Such drugs play antitumor roles by regulating the overall function of the body and have been widely reported as having low toxicity, good efficacy, and high specificity [16]. Some TCM drugs have been used in clinical treatment for cancers such as liver cancer [17].

In our study, we used computational tools and multiple relational databases to explore the pharmacological-action network of S. miltiorrhiza in the treatment of gastric cancer and to predict its active components and potential protein targets and pathways. The Traditional Chinese Medicine System Pharmacology (TCMSP) database and analysis platform is a database that is often used in conjunction with both modern and traditional medicine; it includes 29,384 components, 3311 targets, and 837 related diseases and is helpful for revealing the mechanisms of TCM action [18]. The GeneCards database, including GeneCards (human genes), MalaCards (human diseases), and PathCards (human pathways), has also come to play a key role in clinical research [19]. We searched 18,221 gastric-cancer-related genes in GeneCards.

In this study, we found 102 potential target genes of $S$. miltiorrhiza in the treatment of gastric cancer, mainly involving protein kinase B1 (AKT1), interleukin-6 (IL-6), vascular endothelial growth factor A (VEGFA), epidermal growth factor receptor $(E G F R)$, Fos, mitogen-activated protein kinase 1 (MAPK1), Myc, JUN, Caspase-3 (CASP3), and signal transducer and activator of transcription 3 (STAT3). GO function and KEGG signaling pathway enrichment analyses suggested that the signaling pathway by which $S$. miltiorrhiza exerts its effect in the treatment of gastric cancer is closely related to the PI3K-Akt, HIF-1, and IL-17 signaling pathways.

The PI3K-Akt signaling pathway plays an important role in invasion, proliferation, and apoptosis of gastric-cancer cells. Some studies show that activation of this pathway can enhance invasion and proliferation of such cells [20], as well as inhibit the expression of downstream proteins and promote development of gastric cancer [21]. Inhibiting the PI3K-Akt signaling pathway can induce autophagy of gastric-cancer cells [22]. A chronically hypoxic environment greatly promotes the proliferation, migration, and invasion of these cells [23]. In a hypoxic environment, AKT1 expression was upregulated in cells downstream of the PI3K-Akt signaling pathway, while VEGF expression was upregulated, both of which processes were involved in tumor neovascularization, enhanced cell tolerance to hypoxic environment, and promoted tumor formation [24]. Therefore, activation of the
PI3K-Akt or HIF-1 signaling pathway is an important mechanism for the development of gastric cancer. Inflammation has been reported to be associated with gastriccancer development. IL-6 is a common cytokine that is closely related to gastric-cancer pathogenesis. Cytokines such as adipocytokines can mediate the IL-17 signaling pathway and become important factors in tumor development [25]. Inflammatory factors can play direct roles in immunosuppression, stimulate angiogenesis in gastric cancer, and promote tumor cell formation. CASP3 is a gene that controls apoptosis. Apoptosis is controlled by many intracellular or extracellular signals, including Caspase and B-cell lymphoma 2 (Bcl-2) family proteins. The literature shows that CASP3 is involved in the occurrence and development of gastric cancer, inhibiting the proliferation of gastric-cancer cells and promoting apoptosis [26]. Mitochondria plays a key role in mediating apoptosis through various apoptotic proteins such as cytochrome c $(M y c)$ [27]. The main functions of $M y c$ are to control cell metabolism and apoptosis and to induce Caspase cascade [28]. Caspase is the main executor of apoptosis [29] as a transcription-related protein, and c-Jun is an important cell cycle regulator [30]. In gastric-cancer patients, transcription activity of c-Jun is significantly enhanced and expression of cyclin D1 is activated, which accelerates the transformation of tumor cells from $\mathrm{G} 0 / \mathrm{G} 1$ to $\mathrm{S}$ phase and promotes the proliferation of tumor cells [31].

It can be seen that $S$. miltiorrhiza is a feasible means of treating gastric cancer, and that its potential mechanisms are as follows: (1) inhibiting the PI3K-Akt signaling pathway and regulating proteins downstream thereof so as to inhibit proliferation and promote apoptosis of gastric-cancer cells; (2) reducing the tolerance of gastric-cancer cells to hypoxia in the tumor environment and inducing apoptosis of these cells; and (3) regulating immune system function and inhibiting both inflammation and anti-inflammation in the gastriccancer tumor environment (it should be infiltrated with inflammation, so as to inhibit the local angiogenesis of gastric cancer); (4) activating the CASP3 gene and the activity of apoptotic protease so as to promote apoptosis of gastric-cancer cells; and (5) inhibiting gastric-cancer-related factors so as to inhibit the proliferation of gastric-cancer cells.

Although we have obtained some important new findings based on network pharmacology, there are still limitations to our study. We explored only the feasibility and potential mechanism of $S$. miltiorrhiza in the treatment of gastric cancer at the molecular level, which still needs to be confirmed in the later stage.

In conclusion, this study showed that 65 main active components of S. miltiorrhiza, such as dihydrotanshinone I and miltonones I and II, could be used in the treatment of gastric cancer. These active components could play a pharmacological role in such treatment by acting on the target genes AKT1, IL-6, VEGFA, EGFR, Fos, MAPK1, Myc, Jun, CASP 3, and STAT3, as well as by regulating signaling pathways such as PI3K-Akt, HIF-1, and IL-17. However, 
this study provided only a preliminary prediction of the molecular mechanism by which S. miltiorrhiza treats gastric cancer, and did so at the molecular level of network pharmacology. It lacked further verifications such as polymerase chain reaction testing. In the future, we will do more in-depth research.

\section{Conclusion}

Based on network pharmacology, S. miltiorrhiza is expected to be mined as a candidate Traditional Chinese Medicine (TCM) for the treatment of gastric cancer. Its mechanism for treating this cancer operates via multiple components and pathways. This study provides the basic theory and the basis for further research.

\section{Data Availability}

The original data used to support this study can be available from the corresponding author upon request.

\section{Conflict of Interest}

The authors declare that they have no competing interests.

\section{Acknowledgements}

We thank LetPub for its linguistic assistance during the preparation of this manuscript. At the same time, we also thank Dr. Wang pan, who works in the Zhuhai Financial Work Bureau, P. R. China, for funding this project.

\section{References}

[1] D. Yang, X. Wang, W. Yuan, and Z. Chen. "Intake of Anthocyanins and Gastric Cancer Risk: A Comprehensive Meta-Analysis on Cohort and Case-Control Studies," J Nutr Sci Vitaminol (Tokyo), vol. 65, no. 1, 2019, pp. 72-81.

[2] D. D. Xiong, C. M. Zeng, L. Jiang, D. Z. Luo, and G. Chen. "Ki-67/MKI67 as a Predictive Biomarker for Clinical Outcome in Gastric Cancer Patients: an Updated Metaanalysis and Systematic Review involving 53 Studies and 7078 Patients," J Cancer, vol. 10, no. 22, 2019, pp. 5339-5354

[3] S. Yin, P. Wang, X. Xu, Y. Tan, J. Huang, and H. Xu. "The optimal strategy of multimodality therapies for resectable gastric cancer: evidence from a network meta-analysis," $J$ Cancer, vol. 10, no. 14, 2019, pp. 3094-3101.

[4] B. Zhao, J. Zhang, J. Zhang, et al. "The Impact of Preoperative Underweight Status on Postoperative Complication and Survival Outcome of Gastric Cancer Patients: A Systematic Review and Meta-analysis," Nutr Cancer, vol. 70, no. 8, 2018, pp. 1254-1263.

[5] C. Zhang, W. Hou, J. Huang, et al. "Effects of metastasectomy and other factors on survival of patients with ovarian metastases from gastric cancer: a systematic review and metaanalysis," J Cell Biochem, vol. 120, no. 9, 2019, pp. 1448614498.
[6] D. Zhu, X. Shi, S. Nicholas, Q. Bai, and P. He. "Impact of China's healthcare price reforms on traditional Chinese medicine public hospitals in Beijing: an interrupted timeseries study," BMJ Open, vol. 9, no. 8, 2019, p. e029646.

[7] J. Zheng, G. Li, and Q. Sun. "Misuse of traditional Chinese medicine may be a risk factor to tumorigenesis and progression of head and neck carcinoma in China: a hypothesis based on a case series," J BUON, vol. 24, no. 3, 2019, pp. 1296-1300.

[8] D. D. Xiong, Y. Qin, W. Q. Xu, et al. "A Network Pharmacology-Based Analysis of Multi-Target, MultiPathway, Multi-Compound Treatment for Ovarian Serous Cystadenocarcinoma," Clin Drug Investig, vol. 38, no. 10, 2018, pp. 909-925.

[9] X. Zhang, D. Wang, X. Ren, A. G. Atanasov, R. Zeng, and L. Huang. "System Bioinformatic Approach Through Molecular Docking, Network Pharmacology and Microarray Data Analysis to Determine the Molecular Mechanism Underlying the Effects of Rehmanniae Radix Praeparata on Cardiovascular Diseases," Curr Protein Pept Sci, vol. 20, no. 10, 2019, pp. 964-975.

[10] H. Zhang, Z. Y. Yan, Y. X. Wang, et al. "Network pharmacology-based screening of the active ingredients and potential targets of the genus of Pithecellobium marthae (Britton \& Killip) Niezgoda \& Nevl for application to Alzheimer's disease," Nat Prod Res, vol. 33, no. 16, 2019, pp. 2368-2371.

[11] J. Xue, Y. Shi, C. Li, and H. Song. "Network pharmacologybased prediction of the active ingredients, potential targets, and signaling pathways in compound Lian-Ge granules for treatment of diabetes," J Cell Biochem, vol. 120, no. 4, 2019, pp. 6431-6440.

[12] C. C. Su. "Tanshinone IIA decreases the migratory ability of AGS cells by decreasing the protein expression of matrix metalloproteinases, nuclear factor kappaB-p65 and cyclooxygenase-2," Mol Med Rep, vol. 13, no. 2, 2016, pp. 1263-1268.

[13] T. Wang and X. Fu. "Danshen Formulae for Cancer: A Systematic Review and Meta-Analysis of High-Quality Randomized Controlled Trials". Evid Based Complement Alternat Med, vol. 2019, p. 2310639. https://doi.org/10.1155/2019/2310639.

[14] J. Liu, J. J. Mao, X. S. Wang, and H. Lin. "Evaluation of Traditional Chinese Medicine Herbs in Oncology Clinical Trials," Cancer J, vol. 25, no. 5, 2019, pp. 367-371.

[15] Y. Li, Z. Liu, J. Li, and M. Wang. "Traditional Chinese medicine, Kami-Shoyo-San protects ketamine-induced neurotoxicity in human embryonic stem cell-differentiated neurons through activation of brain-derived neurotrophic factor," Neuroreport, vol. 30, no. 16, 2019, pp. 1102-1109.

[16] H. Y. Zhang, H. L. Wang, G. Y. Zhong, and J. X. Zhu. "Molecular mechanism and research progress on pharmacology of traditional Chinese medicine in liver injury," Pharm Biol, vol. 56, no. 1, 2018, pp. 594-611.

[17] C. Liu, S. Yang, K. Wang, et al. "Alkaloids from Traditional Chinese Medicine against hepatocellular carcinoma," Biomed Pharmacother, vol. 120, 2019, p. 109543.

[18] J. Ru, P. Li, J. Wang, et al. "TCMSP: a database of systems pharmacology for drug discovery from herbal medicines," $J$ Cheminform, vol. 6, 2014, p. 13. 
[19] N. Rappaport, S. Fishilevich, R. Nudel, et al. "Rational confederation of genes and diseases: NGS interpretation via GeneCards, MalaCards and VarElect," Biomed Eng Online, vol. 16, no. Suppl 1, 2017, p. 72.

[20] Y. S. Qiu, G. J. Liao, and N. N. Jiang. "REG3A overexpression suppresses gastric cancer cell invasion, proliferation and promotes apoptosis through PI3K/Akt signaling pathway," Int J Mol Med, vol. 41, no. 6, 2018, pp. 3167-3174.

[21] J. Chen, G. Q. Li, L. Zhang, et al. "Complement C5a/C5aR pathway potentiates the pathogenesis of gastric cancer by down-regulating p21 expression," Cancer Lett, vol. 412, 2018, pp. 30-36.

[22] D. Qiao, Y. Li, J. Xing, et al. "[Baicalein inhibits PI3K/AKT signaling pathway and induces autophagy of MGC-803 cells]," Xi Bao Yu Fen Zi Mian Yi Xue Za Zhi, vol. 35, no. 7, 2019, pp. 613-618.

[23] H. Qiu, G. Hu, and H. Xiong. "Establishment and characterization of chronic-hypoxia-resistant gastric cancer cell line MNK45/HYP," J Huazhong Univ Sci Technolog Med Sci, vol. 31, no. 1, 2011, pp. 52-57.

[24] Y. Kato, M. Yashiro, Y. Fuyuhiro, et al. "Effects of acute and chronic hypoxia on the radiosensitivity of gastric and esophageal cancer cells," Anticancer Res, vol. 31, no. 10, 2011, pp. 3369-3375.

[25] L. F. Dong, S. Y. Xu, J. P. Long, F. Wan, and Y. D. Chen. "RNA-Sequence Analysis Reveals Differentially Expressed
Genes (DEGs) in Patients Exhibiting Different Risks of Tumor Metastasis," Med Sci Monit, vol. 23, 2017, pp. 2842-2849.

[26] C. Y. Zhu, F. Q. Meng, and J. Liu. "MicroRNA-524-5p suppresses cell proliferation and promotes cell apoptosis in gastric cancer by regulating CASP3," Eur Rev Med Pharmacol Sci, vol. 23, no. 18, 2019, pp. 7968-7977.

[27] T. P. Xu, P. Ma, W. Y. Wang, et al. "KLF5 and MYC modulated LINC00346 contributes to gastric cancer progression through acting as a competing endogeous RNA and indicates poor outcome," Cell Death Differ, vol. 26, no. 11, 2019, pp. 2179-2193.

[28] L. O. Lopes, J. H. Maues, H. Ferreira-Fernandes, et al. "New prognostic markers revealed by RNA-Seq transcriptome analysis after MYC silencing in a metastatic gastric cancer cell line," Oncotarget, vol. 10, no. 56, 2019, pp. 5768-5779.

[29] Y. Yuan, Z. Ding, J. Qian, et al. "Casp3/7-Instructed Intracellular Aggregation of $\mathrm{Fe}_{3} \mathrm{O}_{4}$ Nanoparticles Enhances T2 MR Imaging of Tumor Apoptosis," Nano Lett, vol. 16, no. 4, 2016, pp. 2686-2691.

[30] Z. Wang, F. Zou, Y. Tian, B. Xiang, B. Qin, and Y. Liu. "Paclitaxel reversed trastuzumab resistance via regulating JUN in human gastric cancer cells identified by FAN analysis," Future Oncol, vol. 14, no. 26, 2018, pp. 2701-2712.

[31] P. Chen, X. Luo, Z. Che, et al. "Targeting of the C-Jun/BCL$\mathrm{XL} / \mathrm{P} 21$ Axis Accelerates the Switch from Senescence to Apoptosis Upon ROC1 Knockdown in Gastric Cancer Cells," Cell Physiol Biochem, vol. 48, no. 3, 2018, pp. 1123-1138. 